УДК 004.9

https://doi.org/10.36906/AP-2020/21

\title{
ОСНОВЫ СОЗДАНИЯ ПАНОРАМ ДЛЯ РЕАЛИЗАЦИИ ВИРТУАЛЬНОЙ ЭКСКУРСИИ
}

\author{
Пащенко А. И. \\ Нижневартовский государственный университет \\ г. Нижневартовск, Россия \\ Манюкова Н. В. \\ канд. пед. наук \\ Нижневартовский государственный университет \\ 2. Нижневартовск, Россия
}

\begin{abstract}
Аннотация. В статье рассмотрены возможности применения панорам в области виртуальных экскурсий. Проанализированы требования создания качественной панорамы. Представлены результаты исследования в качестве готовой панорамы для последующей реализации виртуальной экскурсии.
\end{abstract}

Ключевые слова: панорама, проектирование панорам, виртуальная экскурсия, проектирование виртуальной экскурсии, цилиндрические панорамы, работа в PTGui.

Актуальность виртуальных экскурсий заключается в возможности принимать участие в просмотре, прогулке и не только. Это намного интереснее пассивного наблюдения. Данная возможность дает яркие впечатления и наиболее четкое преставление о том, с чем имеешь дело, ведь в отличие от виртуальной экскурсии, просматривая обычную фотографию или видео, зритель видит только то, что ему показывают, и не может управлять процессом просмотра.

Результаты исследований в сфере взаимосвязи компьютерных технологий и людей с ограниченными возможностями здоровья показали, что виртуальные экскурсии эффективны для передачи информации. Людям с физическими недостатками трудно путешествовать, поэтому виртуальная экскурсия - хороший инструмент, который может помочь им.

К тому же, в данный момент времени, когда соблюдается режим самоизоляции, идея этого проекта позволяет людям проводить свободное время дома, путешествуя с помощью виртуальных экскурсий по различным достопримечательностям всего мира, и при этом, не нарушая условия карантина.

Как правило, фотопанорамы формируются из нескольких заранее подготовленных фотографий, которые затем сшиваются при помощи специальных программ в единую панораму. В фотоаппарате должна быть учтена функция фиксации экспозиции - ручной режим установки выдержки и диафрагмы, а также ручной режим установки баланса белого, благодаря чему какая-либо фотография не будут отличаться от другой контрастностью и яркостью. Для съемки будет использоваться цифровая видеокамера Sony FDR-AX33. Целесообразнее использовать фотоаппарат, но уже имеется достойная видеокамера, которая полностью отвечает перечисленным выше требованиям [1].

Одним из весомых моментов создания панорамы - необходимость вращения видеокамеры вокруг нодальной точки объектива. Нодальная точка - точка внутри объектива камеры, где пересекаются лучи света, идущие к матрице. При вращении 
видеокамеры вокруг этой самой точки отсутствует параллакс объектов. Параллакс смещение объектов фронтального плана сравнительно с объектами заднего плана при повороте видеокамеры. Это смещение может вызвать проблемы при сшивании панорам. Чтобы избежать подобных трудностей будет использован штатив с панорамной головкой.

Фотографировать каждый дальнейший кадр стоит так, чтобы он перекрывал предшествующий примерно на 30-35\%. Больший процент перекрытия означает лучшее качество сборки готовой фотопанорамы, благодаря большему количеству похожих объектов. Необходимо следить, чтобы не изменялась линия горизонта, контролировать это позволяют пузырьковые уровни, которые располагаются на штативе. Кроме того, места швов лучше располагать на довольно однотонных объектах.

При фотографировании важно, чтобы не попадали движущиеся объекты в кадр, например, люди, облака, транспорт, качающиеся от ветра элементы, и необходимо, чтобы не изменялось освещение. Так же важно заранее обдумать точки съемки (рис. 1).

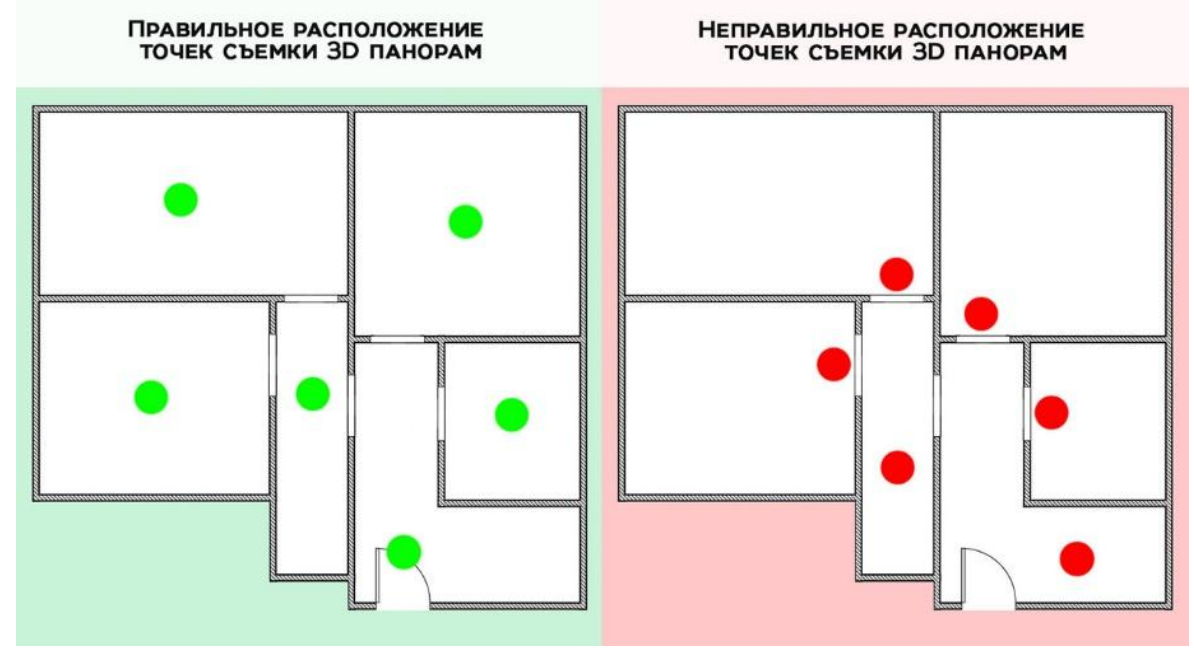

Рис. 1. Расположение точек съемки

Применение автофокусировки при съемке 3D-панорамы возможно приведет к тому, что отдельные кадры могут сфокусироваться по-разному, или, что намного хуже, некоторые кадры могут вовсе оказаться вне фокуса, в таком случае собрать панораму будет невозможно (https://clck.ru/T9Yw5).

Сшивание фотографий в виртуальные панорамы производится в специально предназначенных для этого программах, обычно, в которых можно сшивать в автоматическом, полуавтоматическом и ручном режимах. Поскольку будет использоваться штатив, и угол наклона видеокамеры не изменится, можно включить автоматический режим, так как необходимости указывать контрольные точки на всех парах фотографий нет, это потребуется сделать лишь на нескольких снимках для наиболее хорошей панорамы.

Потом будет производиться состыковка обработанных изображений между собой. Автоматически или вручную путем установки контрольных точек для каждой пары граничащих фотографий. Последнее достаточно кропотливо, но зачастую можно совместить далеко не идеальные кадры. Конечно, качество сшивания фото зависит от точности установки контрольных точек. Затем, на финальном этапе соседние изображения смешиваются для выравнивания их яркости и контрастности. 


\section{СОВРЕМЕННОЕ ПРОГРАММИРОВАНИЕ}

III Международная научно-практическая конференция

Итогом данных действий будет созданная панорама. Для того, чтобы преобразовать ее в виртуальную нужно конвертировать фотопанораму в подходящий формат, а для презентации виртуальной панорамы в сети ее потребуется вручную встроить в файл существующей web-страницы либо сгенерировать шаблонную интернет-страницу (http://habrahabr.ru/qa/4885).

Программа PTGui (продукт нидерландской компании New House Internet Services BV) считается одной из лучших в среде программ, предназначенных для сшивания картинок. Изначально программа задумывалась как интерфейс для популярной среды создания фотопанорам Panorama Tools. Затем, с версии 5.0, программа стала использоваться и как дополнение для вышеуказанного приложения, и как отдельная программа для создания фотопанорам.

PTGui может автоматически ставить контрольные точки, сглаживать горизонт, проводить коррекцию цвета и экспозиции. Так же существует функция построения многорядных панорам и больших гигапиксельных фото. Результаты работы можно сохранять в виде многослойной картинки. Дальше ее удобно обрабатывать в фотошопе. Имеется поддержка HDR. На скриншоте показано окно приложения (рис. 2).

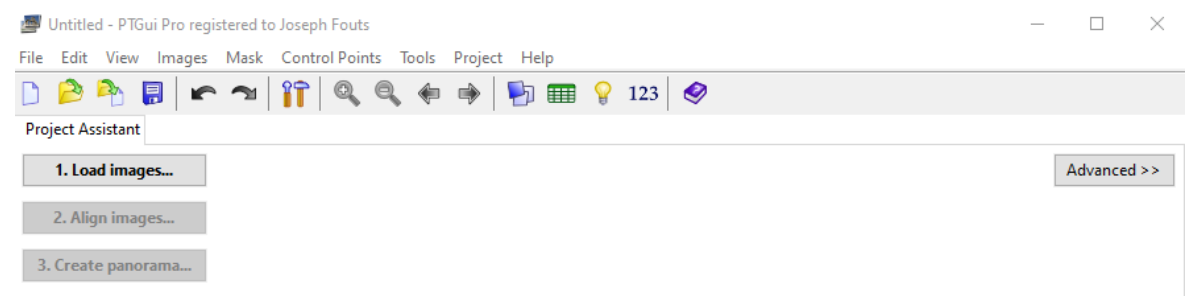

Рис. 2. Окно «PTGui»

Проводя работу в простом режиме, фотопанорама создается за три шага: загрузка фотографий, анализ фото, создание панорамы.

После съемки нужных локаций, можно будет перейти к созданию панорамы. Начинаем с загрузки фото в PTGui (рис. 3). Сделать это можно нажатием соответствующей кнопки и указав на диске заранее подготовленные фотографии или просто перетаскиванием нужных файлов в окно программы.

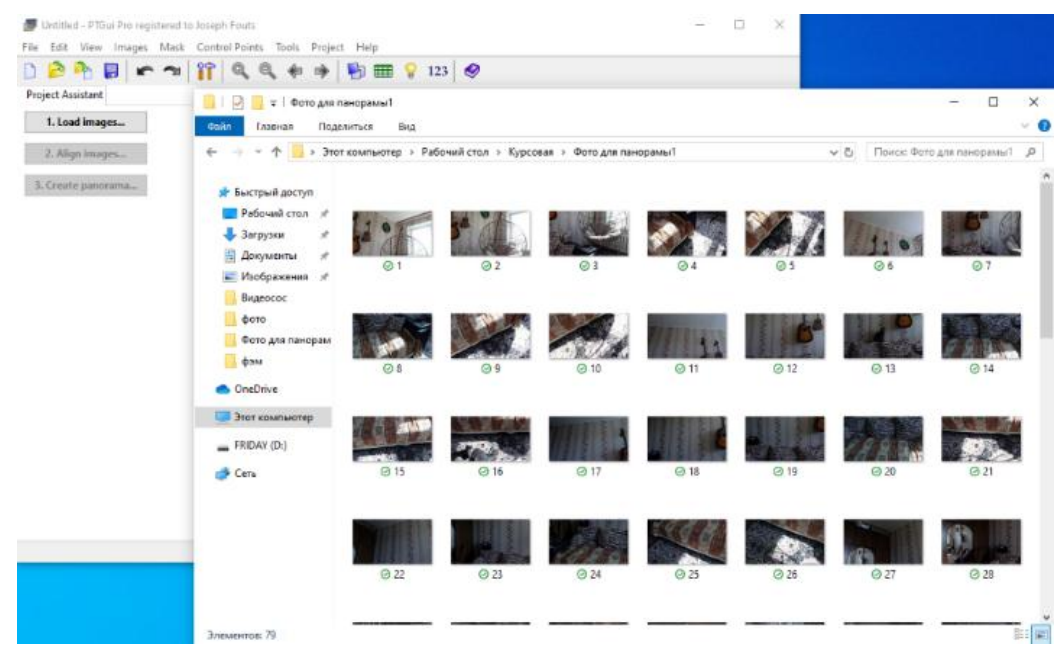

Рис. 3. Загрузка снимков в "PTGui" 


\section{СОВРЕМЕННОЕ ПРОГРАММИРОВАНИЕ}

\section{III Межкународная научно-практическая конференция}

В программе фотографии будут отображаться как вертикальная лента (рис. 4). Нажав на ленту, появится окно Source Images. В нем можно установить порядок размещения изображений.

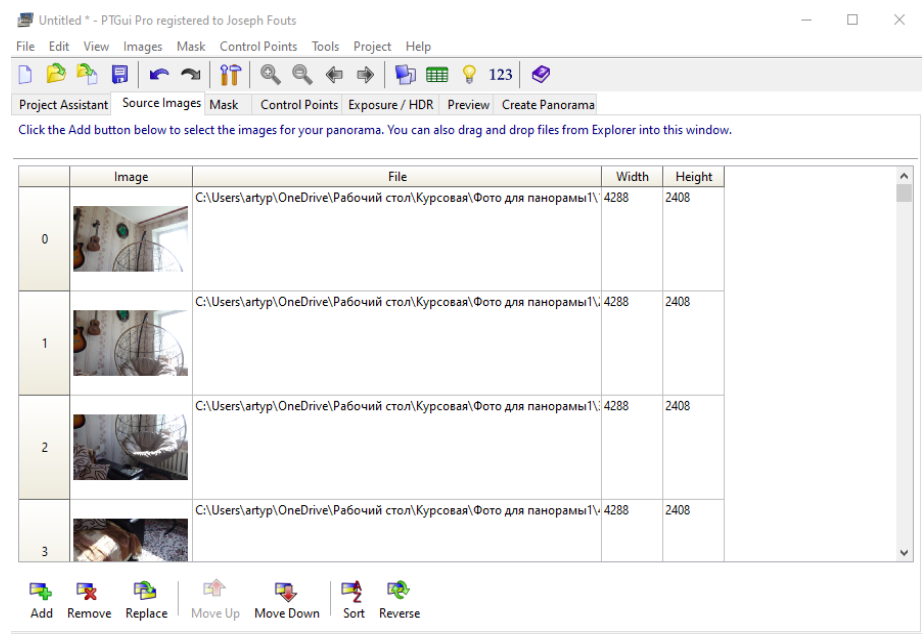

Рис. 4. Лента с фотографиями в "PTGui"

Дальше жмем кнопку “Align images”. Начнется анализ загруженных фотографий, после чего в перекрывающихся зонах смежных снимков создаст контрольные точки. Далее PTGui склеит фото и оптимизирует их. После того, как программа произведет автоматическое выравнивание, на экране откроется окно Panorama Editor (рис. 5). Здесь можно менять ориентацию полной панорамы и отдельных ее частей. Двигая ползунок сбоку, можно обрезать ненужные в панораме объекты.

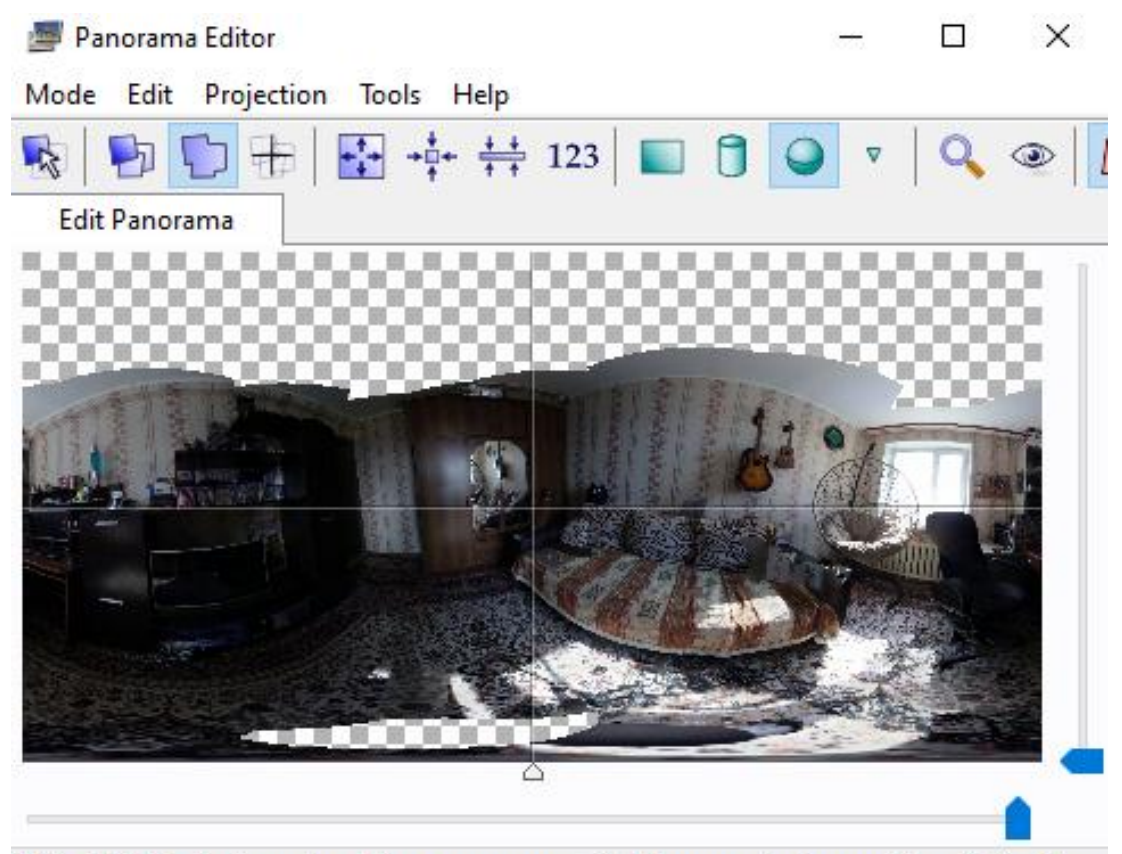

$360^{\circ} \times 180^{\circ}$ Equirectangular - Move panorama with left mouse button, rotate with right $\mathrm{k}$

Рис. 5. Окно "Panorama Editor"

Порой программа не совсем правильно определяет места склеивания изображений, иногда нужно вручную сшивать фотографии, добавляя дополнительные контрольные точки. 
Контрольные точки - пары отметок на сшиваемых картинках. Они обозначают одинаковые объекты на фотографиях. Чем точнее указаны контрольные точки и чем больше их указать, тем незаметнее получится шов между снимками. Чтобы управлять контрольными точками изображений нужно перейти на вкладку Control Points. Ниже представлены сшиваемые кадры, на которых находятся пары контрольных точек (рис. 6). Точки имеют нумерацию для удобства. Так же каждая пара точек имеет свой цвет для более легкого ориентирования. Под снимками находится таблица с подробной информацией о координатах контрольных точек с обеих сторон.

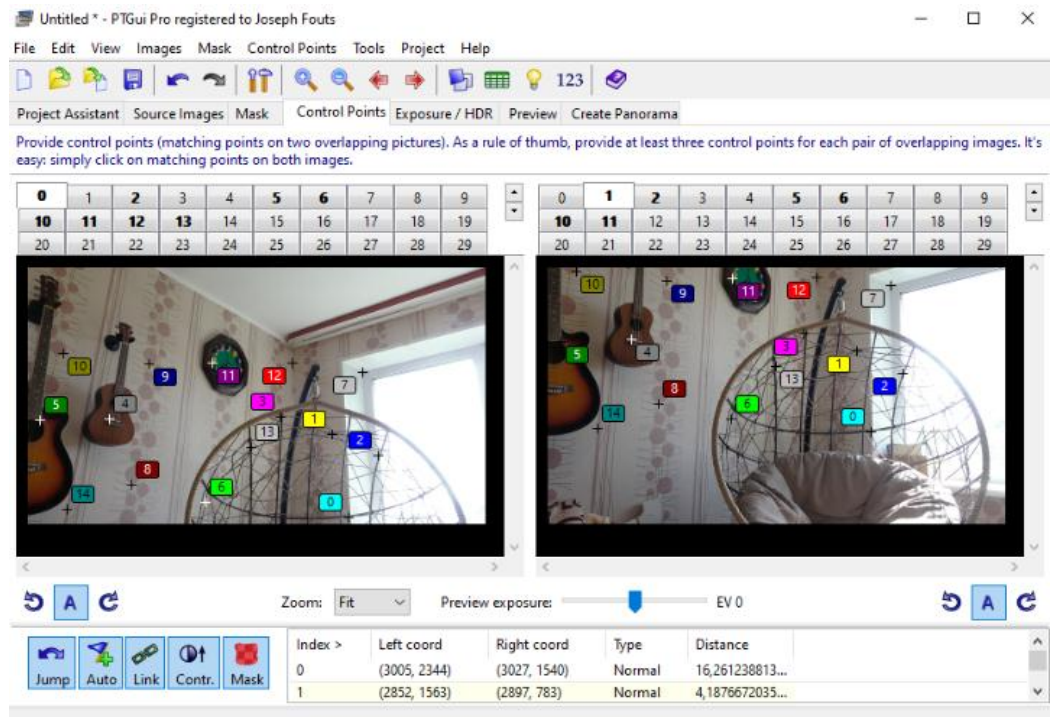

Рис. 6. Меню контрольных точек

Контрольные точки иногда определяются не совсем верно, ведь алгоритмы программы не идеальны. Если такое случилось, стоит щелкнуть правой кнопкой мыши на неверной точке, а затем удалить неудачную отметку командой “Delete”.

Потом можно поставить контрольные точки вручную, нажимая по снимкам на объектах, которые есть слева и справа. Парная контрольная точка будет указана самостоятельно, но нужно будет проследить, правильно ли ее поставили алгоритмы. Если она указана не совсем верно, нужно передвинуть ее на правильное место. Далее надо установить тем же способом максимальное количество контрольных точек, пытаясь, расставить их не только в одном месте, а по всей перекрывающейся поверхности снимков. Также очень важно размещать контрольные точки с максимальной точностью.

Для идеальной склейки без видных швов, расстояние между парами контрольных точек должно быть, как можно меньше. Во время оптимизации рассчитывается, каким образом должны трансформироваться и выравниваться кадры панорамы, чтобы создать минимальную дистанцию между контрольными точками (https://clck.ru/T7hoC).

Чтобы это сделать нужно открыть вкладку Optimizer, перед этим нажав Advanced на вкладке Project Assistant для того чтобы перейти в расширенный режим. В списке коррекции дисторсии линзы выбрать опцию "Heavy + lens shift". Выбираем алгоритм оптимизации PTGui, запускаем процесс оптимизации, затем будет показано окно с результатами, в котором представлены минимальное, среднее и максимальное расстояние между контрольными точками. Нужно сделать как можно меньше среднюю дистанцию между контрольными точками. Помимо цифр в результатах оптимизации PTGui предоставляет еще одну оценку проведенного процесса — "very bad", "bad", "not so bad", "not so good", "good", 
"very good" или "too good to be true". Однако, полагаться только на данные оценки не нужно, так как размер исходных фотографий не учитывается.

Лучше уменьшить среднее расстояние, если оно выходит больше 20 ріх. Для этого нужно подтвердить оптимизацию и вернуться к таблице контрольных точек. Следует проверить, что контрольные точки были упорядочены по расстоянию. Если будет показано, что некоторые точки сильно отклонены от среднего значения, то их стоит удалить. Далее вновь выполняем оптимизацию и смотрим результат. Желательно повторить удаление контрольных точек с плохими значениями расстояния и оптимизацию, пока не будет достигнут хороший результат. Однако, нужно следить, чтобы оставалось достаточное количество контрольных точек для склеивания панорамы и не показались дефекты.

В процессе оптимизации получили среднее расстояние 19.52 ріх, такой результат получил оценку “very good” (рис. 7). Этот результат нам подходит, можем двигаться дальше.

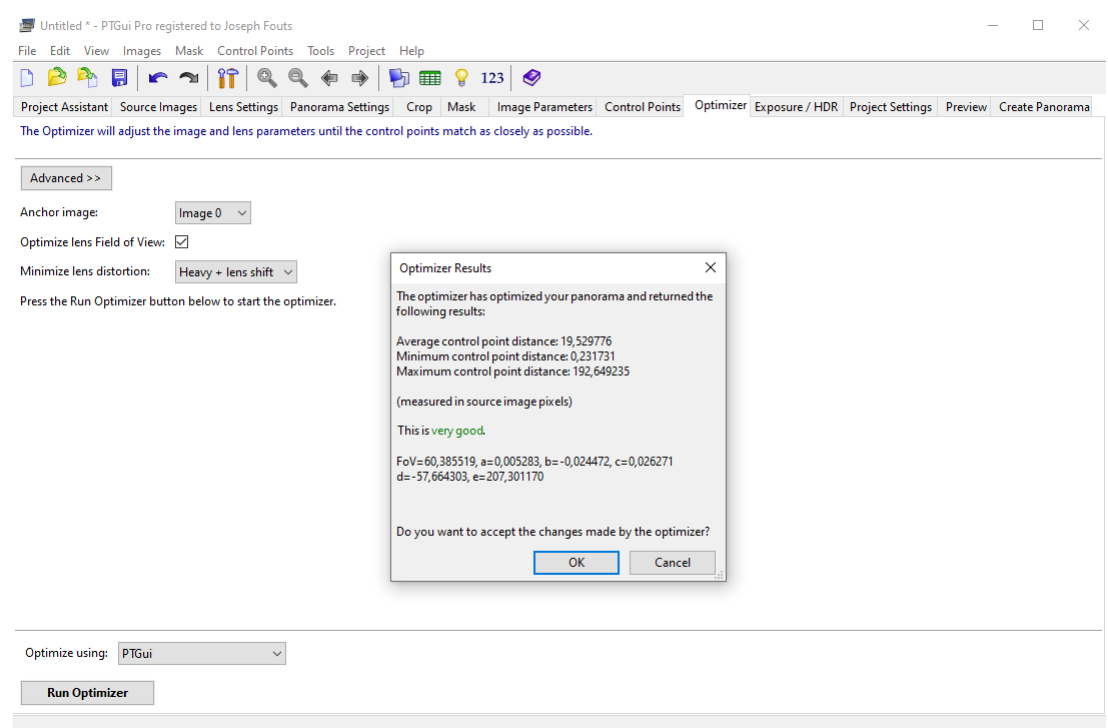

Рис. 7. Результат оптимизации

После того, как мы выполнили оптимизацию, переходим на закладку Create Panorama. Тут можно задать путь сохранения и имя файла, выбрать формат панорамы нужный размер. Меню Create Panorama представлено ниже (рис. 8).

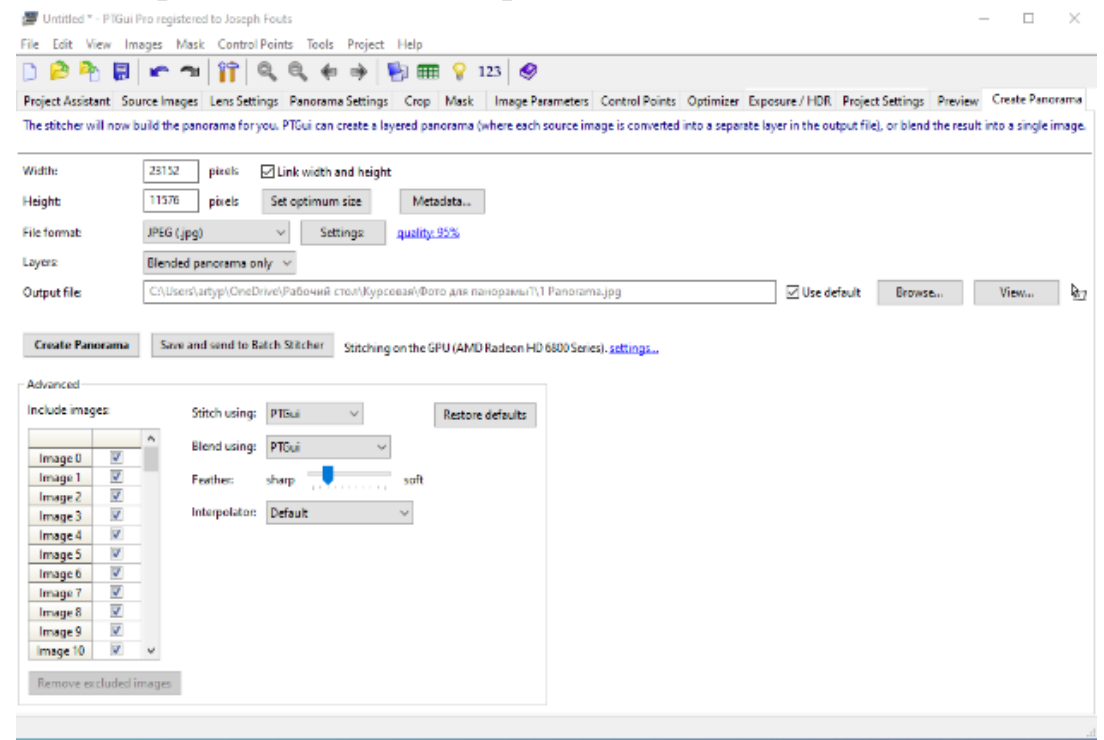

Рис. 8. Меню Create Panorama 


\section{СОВРЕМЕННОЕ ПРОГРАММИРОВАНИЕ}

III Международная научно-практическая конференция

B PTGui Pro можно сохранять панораму в виде отдельных слоев, где каждый из слоев соответствует каждому начальному снимку панорамы. Данная функция может быть очень полезной, если необходимо отретушировать на панораме повторяющиеся движущиеся объекты.

И вот, начинаем процесс создания панорамы и ждем окончания. Процесс может продолжаться от считаных секунд до нескольких часов. Все зависит мощности ПК, размера снимков, их количества и размера итоговой панорамы.

Получаем проекцию цилиндрической панорамы. Результат представлен ниже (рис. 9).

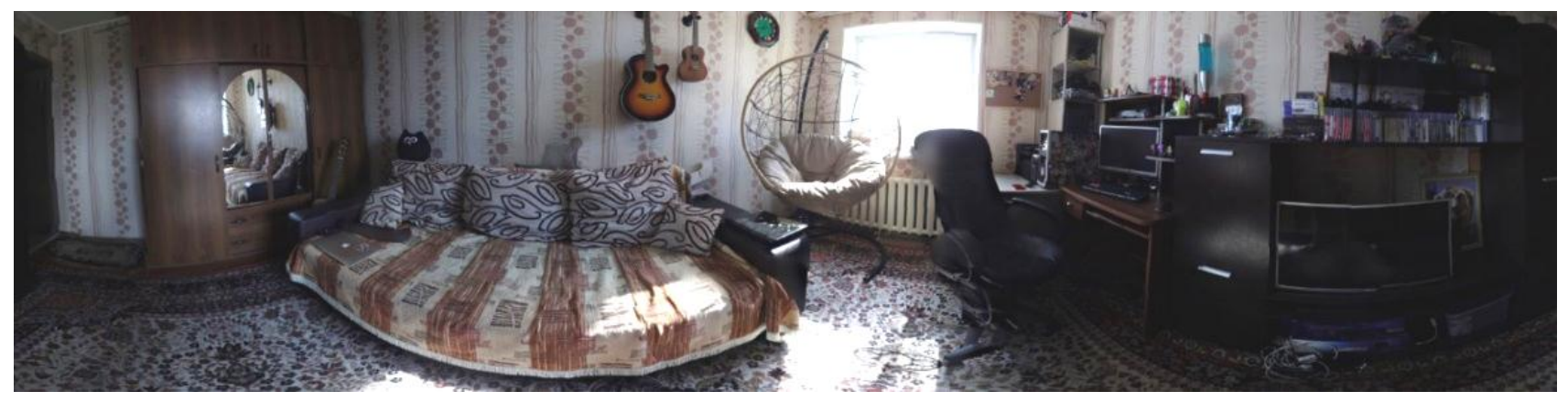

Рис. 9. Цилиндрическая панорама (https://clck.ru/T7hpd)

В итоге - готовая панорама, которая может использоваться для создания виртуальной экскурсии.

\section{Литература}

1. Лапин А.И. Фотография как... М.: Издательство МГУ, 2003.

СПащенко А. И., Манюкова Н. В., 2020 